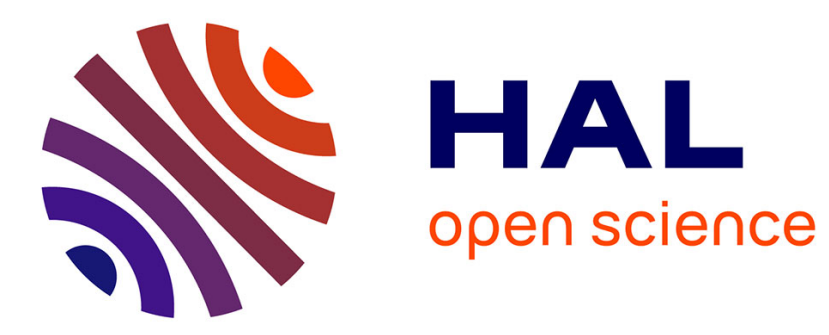

\title{
Information-driven coordination: experimental results with heterogeneous individuals
}

Viktoriya Semeshenko, Alexis Garapin, Bernard Ruffieux, Mirta B. Gordon

\section{To cite this version:}

Viktoriya Semeshenko, Alexis Garapin, Bernard Ruffieux, Mirta B. Gordon. Information-driven coordination: experimental results with heterogeneous individuals. 2009. hal-00404231

\section{HAL Id: hal-00404231 \\ https://hal.science/hal-00404231}

Preprint submitted on 15 Jul 2009

HAL is a multi-disciplinary open access archive for the deposit and dissemination of scientific research documents, whether they are published or not. The documents may come from teaching and research institutions in France or abroad, or from public or private research centers.
L'archive ouverte pluridisciplinaire HAL, est destinée au dépôt et à la diffusion de documents scientifiques de niveau recherche, publiés ou non, émanant des établissements d'enseignement et de recherche français ou étrangers, des laboratoires publics ou privés. 


\title{
Information-driven coordination: experimental results with heterogeneous individuals
}

\author{
Viktoriya Semeshenko ${ }^{(1,3)}$, Alexis Garapin ${ }^{(2)}$ \\ Bernard Ruffieux ${ }^{(2)}$, Mirta B. Gordon ${ }^{(1)}$ \\ (1) Laboratoire TIMC-IMAG (UMR 5525), University of Grenoble I \\ Domaine de La Merci - Jean Roget, 38706 La Tronche, France, \\ (2) University of Grenoble II, UMR 1215 GAEL, F-38000 Grenoble, France, \\ (3) Departamento de Economía, Facultad de Ciencias Económicas, \\ Universidad de Buenos Aires, Av. Córdoba 2122 - 1120 AAQ Buenos Aires, Argentina
}

May 2, 2009

\begin{abstract}
We study experimentally a coordination game with $\mathrm{N}$ heterogeneous individuals under different information treatments. We explore the effects of information on the emergence of Pareto-efficient outcomes, by means of a gradual decrease of the information content provided to the players in successive experiments. We observe that successful coordination is possible with private information alone, although not on a Pareto-optimal equilibrium. Reinforcement-based learning models reproduce the qualitative trends of the experimental results.
\end{abstract}

\section{Introduction}

Most of the coordination games described in the literature are restricted to homogeneous players. However, players are likely to have heterogeneous preferences; in this case equilibria cannot be simply extrapolated from the individual players' intentions by introspection [Granovetter(1978), Schelling(1978)]. In a decentralized environment, information provided to the players may help to coordinate their choices. Coordination mechanisms proposed in the experimental literature (see [Devetag and Ortmann(2007)]) include pre-play communication [DeJong et al.(1994)], cheap talk [Farell(1987)], repetition [Crawford and Haller(1990)], and learning in repeated games -

[Kandori et al.(1993)].

In this paper we consider the dying seminar, a coordination game with heterogeneous individuals introduced by [Schelling(1978)], to explore the effects of information available to players on coordination. This game represents a situation in which collective activities are driven by 
personal motivations. A group of researchers eager to meet regularly organize a weekly seminar. Each potential participant has his idiosyncratic preference for attending the seminar. Even if the first meeting has a good attendance, frequently the number of persons in successive meetings drops and eventually the seminar "dies". Usually most of the potential participants regret the outcome, claiming that they would have attended if enough other participants had attended regularly. Assessing this enough number introduces interactions among individuals and leads to coordination problems arising from possible multiple equilibria. The Pareto-optimal equilibrium may be reached if a critical mass of individuals choose to participate.

The dying seminar arises in many everyday life circumstances, where social or economic activities are considered interesting or worthwhile only if enough individuals participate in them. Examples include the stratification of investment in human capital and economic segregation [Benabou(1996)], the large dispersion of urban crime in similar cities [Glaeser et al.(1996)], the emergence of social norms [Ostrom(2000)], the choice of a computer operating system [Katz and Shapiro(1994)], the smoking behavior [Krauth(2006), Soetevent and Kooreman(2007)], the persistence in the educational level within neighborhoods [Durlauf(1996)] or the subscription to a telephone network [Artle and Averous(1973), Rohlfs(1974), Rabenau and Stahl(1974), Curien et al.(1987)] among others.

In our experiment, the game is played a finite number of periods under various information treatments. We progressively decrease the information provided to the subjects in successive experiments, in order to study the dependence of the coordination level reached on the information available.

The results obtained show that knowing the actual number of participants in the preceding periods is enough information to coordinate the individual choices on the Pareto-optimal equilibrium. Furthermore, in contrast with earlier experimental evidence [Van Huyck et al.(1990), Van Huyck et al.(1997), Berninghaus and Ehrhart(2001), Devetag(2003)], we observe that even with private information alone successful coordination is possible, although not on the Paretooptimal equilibrium.

The paper is organized as follows: the game and its equilibrium properties are presented in section 2 . We shortly review the relevant literature in section 3 . The experimental design and procedures are described in section 4 . The results are presented and discussed in section 5 . We propose a learning model that allows us to interpret the observed behavior in section 6 . We conclude in section 7 .

\section{Theoretical model with heterogeneous individuals}

We consider a group of $N$ potential participants of the seminar. Following Schelling, we make the simplifying hypothesis that each individual's decision is only conditioned by the actual number of participants, $P$. As explained in section 4, our experimental setup allows to implement this hypothesis explicitly. We assume that each individual $i(1 \leq i \leq N)$ has a private threshold $H_{i}$, which is the minimum number of participants (including himself) above which he finds it worthwhile to attend the seminar. 
If individual $i$ attends the seminar and $P$ turns out to be larger or equal (less than) to $H_{i}$, his utility is large (small). If he does not participate his utility has an intermediate value. In the experiment utilities are translated into payoffs $\pi_{i}$.

We assume that the thresholds $H_{i}$ are idiosyncratic characteristics of the agents that remain constant during the experiment. We thus exclude the possibility that in repeated iterations the agents adapt their values of $H_{i}$ to the successive outcomes. These thresholds may have any integer value between 0 and $N+1$. A threshold of $N+1$ means that the individual does not want to participate, whatever the number of participants; conversely, a threshold of 0 or 1 means that the individual is willing to participate independently of the number of participants. Thresholds 0 and 1 are thus non-strategic.

If $i$ decides to attend the seminar, his decision $\omega_{i}$ is set at $\omega_{i}=1$; otherwise $\omega_{i}=0$. The payoff $\pi_{i}$ of agent $i$ depends on his decision through

$$
\pi_{i}=\left\{\begin{array}{lll}
U & \text { if } \quad \omega_{i}=1 \text { and } P \geq H_{i} \\
V & \text { if } \quad \omega_{i}=1 \text { and } P<H_{i} \\
W & \text { if } \quad \omega_{i}=0
\end{array}\right.
$$

where $U>W>V$. Thus, the decision that maximizes the payoff is

$$
\begin{array}{ll}
\omega_{i}=1 & \text { if } P \geq H_{i} \\
\omega_{i}=0 & \text { if } P<H_{i}
\end{array}
$$

Before considering how individuals make their decisions, we analyze the equilibria of this model. To this end we do not need to know each individual's threshold, but only the proportion of individuals that have each threshold value, i.e. the probability mass function (pmf) $f(H)$. The corresponding cumulative distribution, $F(H)$, represents the fraction of individuals that have a threshold $H$ or smaller.

Schelling proposed a simple construction leading to the equilibria of the dying seminar game, assuming that the thresholds' cumulative distribution $F(H)$ is a monotonically increasing continuous function. This assumption, pertinent for the study of very large systems, is strictly correct in the infinite population limit. Although infinite and finite systems share some generic properties, finite size systems present some specific characteristics. One of the more conspicuous is that in finite systems $f(H)$ has a discrete support. Schelling's construction, restricted to rational values of $H / N$, is still valid for the corresponding step-wise cumulative $F(H)$ function. Analytically, the properties of finite systems may be deduced from those of the infinite population limit using finite-size corrections. However, a continuous probability density function cannot be used to represent populations where several agents have the same threshold, as is the case in our experiments (see Fig. 1). In the infinite population limit, a threshold shared by several agents (say, by a finite fraction of individuals $\rho$ ) is represented by a Dirac-delta distribution of weight $\rho$ in the probability function, and has to be handled carefully when solving the equilibrium equation. Moreover, pure coordination problems within the sub-group of individuals having the same threshold may hinder the system's convergence to a Nash equilibrium, as is discussed in section 4.1. 
At equilibrium, the number of participants of the seminar, $P$, must be equal to the fraction of individuals whose threshold is less than or equal to $P$. These satisfy:

$$
\frac{P}{N}=F(P)=\sum_{H=H_{\min }}^{P} f(H),
$$

where $F(H)$, the cumulative thresholds' distribution, is the fraction of individuals with threshold $H$, and $H_{\min }$ is the smallest among the individuals' thresholds. The principle of Schelling's graphic construction used to find the solutions of (3) remains the same: these are given by the intersections of $y=N F(H)$ with the diagonal $y=H$ at integer values of $H$. They correspond to the situations that include the $P$ participants whose thresholds satisfy $H_{i} \leq P$.

Equilibria are fixed points, which may be stable (Nash equilibria) or unstable (called "critical masses" in the literature). Notice that only intersections with steps are equilibria: intersections with vertical lines connecting two consecutive steps, that may arise when several individuals have the same threshold value, are not equilibria, though they may still be considered as critical masses as is explained in the description of the experimental seminars (see section 4.1).

For some distributions $f(H)$ eq. (3) may present several equilibria, raising a potential conflict between efficiency (i.e. selecting a payoff dominant outcome) and security (i.e. following a maxmin strategy, see [Rankin et al.(2000)] for a discussion). This coordination dilemma increases when available information concerning other players' thresholds or behavior is scarce.

\section{Coordination games and information treatments}

The dying seminar is a coordination game with asymmetric equilibria and heterogeneous preferences, and with strategic complementarities [Camerer(2003), Bowles(2004)]. In this section we shortly review the stag-hunt and order-statistics games, which have characteristics similar to the dying seminar, and we point out the main differences. We only look at experiments that share our goal, namely to study the rôle of available information on the outcome of iterated coordination games. In contrast to the dying seminar game, heterogeneity in the individual preferences is not explicitly incorporated in the stag hunt and order-statistics games considered in the literature ${ }^{1}$ : behavioral idiosyncratic differences clearly exist, but remain out of the experimentalist's control.

Stag hunt games. The dying seminar is a variant of the stag hunt game, played by $N$ hunters. Both games represent economic situations of strategic complementarities where the Paretodominant equilibrium requires risky decisions. [Cooper et al.(1992)] studied the incidence of

\footnotetext{
${ }^{1}$ Heterogeneity has been included in market entry games [Seale et al.(2002)], which are binary decision problems, like the dying seminar game. One may be tempted to assimilate the market capacities to the number of participants in the seminar. However, these games are of a very different nature. Players' strategies are complements in the dying seminar game, while they are substitutes in the market entry game. In other words, the payoff to participants or entrants $\left(\omega_{i}=1\right)$ is an increasing function of $P$ in the dying seminar (with an idiosyncraticdependent discontinuity at $H_{i}$ ), but a decreasing function of $P$ in market entry games. As a consequence, the equilibria in market entry games do not correspond to the maximization of group payoffs.
} 
information available to players on the stag hunt game's outcome, by allowing for non-binding cheap talk messages. These authors consider a setting where $N$ subjects are randomly paired to play simultaneous $N / 2$ two-person games at each iteration. Thus, each subject's estimation for the next period is based on a random sampling of the choices of his successive partners. Their results suggest that it is difficult to achieve efficiency without any pre-play communication. Allowing for such communication between potential partners before the random pairing increases dramatically the frequency of convergence to the Pareto-dominant equilibrium.

Despite the similarities, there are at least two main differences between both kinds of games. Firstly, the Pareto-dominant equilibrium in the dying seminar game requires simultaneous coordination of a large number of players, and thus it may be more difficult to reach than in the sets of two-person games played in the stag hunt game. Secondly, we expect that the players implement different learning schemes in the two games, due to the different nature of the information obtained at successive periods. Random sampling in the stag hunt game may introduce a large variance in the players' estimations. There is no randomness in the dying seminar game. However, variance in the subjects' behaviour is expected, but mainly due to the heterogeneity introduced by the idiosyncratic thresholds.

Order-statistics games. This class of coordination games is more similar to the dying seminar in that they involve $N(N>2)$ players playing the same game. Each player has to choose a number $i$ and his payoff depends on $i$ and on some order-statistics (e.g. the minimum, the median, the average) of the population's choices. Like the dying seminar, these games admit multiple Nash equilibria, which may or may not be ranked, depending on the structure of the payoff function.

In the reference paper by [Van Huyck et al.(1990)], they consider the "weak link game" where the possible values of $i$ belong to a finite set of numbers and the order-statistics is the minimum. The payoff function is $\pi(i)=a \underline{i}-b i$ where $\underline{i}$ is the minimum among all the players' choices, and $a>b>0$. The best reply for a player to the collective outcome is to choose $i=\underline{i}$. By symmetry, any solution where all the players choose the same number is a Nash equilibrium. The Pareto-dominant equilibrium corresponds to choosing the largest value for $i$. The authors' main objective is to assess whether players will coordinate on the latter. In experiments involving different numbers of subjects, the order-statistics of the actual choices is publicly announced to the players at the end of each period. Results show that subjects mostly coordinate on the most inefficient equilibrium, due to strategic uncertainty. Moreover, the difficulty to coordinate on a Pareto-dominant equilibrium increases with $N$.

[Berninghaus and Ehrhart(2001)] consider the same game under different information treatments. In each period the subjects are informed of either the order-statistics of the actual choices, as in the experiments by [Van Huyck et al.(1990)] (treatment I), or the distribution of the subjects' strategy choices (treatment II), or each opponent subject's individual strategy choice (treatment III). Results under treatment I are similar to those of [Van Huyck et al.(1990)]: subjects mostly coordinate on the secure equilibrium. However, in treatments II and III subjects tend to choose the Pareto-dominant strategy. Their results show that coordination failures can 
be avoided, at least in order-statistics games, provided that subjects have access to information about their opponents' previous choices.

$[\operatorname{Devetag}(2003)]$ considers another order-statistics game, in which players must choose a number $i$ in the range $i \in[0, N]$. If the total number of subjects that choose number $i$ is larger than $i$, they all get the same payoff, proportional to $i$. Choices not satisfying this condition have zero payoff. She finds that convergence to the Pareto-dominant equilibrium $i=N$ is the modal outcome only under complete information conditions, in which the distribution of all individual choices of all preceding periods is provided to all subjects. When only the median of the previous period choices is announced the groups coordinate on inefficient equilibria. No coordination at all is observed when subjects know only their preceding period payoffs. The experiments under complete information conditions also show that introducing increasing returns, through payoffs proportional to the number of subjects choosing the same number $i$, speeds up convergence to the payoff-dominant equilibrium. In fact, increasing returns are an incentive to choose high numbers, and this from the very first period. Such high- $i$ choices act then as signals favoring coordination.

The dying seminar game is close to these order-statistics games in that the individual payoffs depend on the collective outcome involving the choices of a large number of players $(N>>2)$, a fact that may hinder coordination. Like in the weak-link game, the Pareto-dominant equilibrium is optimal because it maximizes the number of winners, but not the payoff of each of them. The main difference with the order-statistics games considered by Devetag lies in the structure of the payoff function. In the dying seminar it does not depend on the size of the self-coordinated groups of winners. Since the payoff is the same for all the winners, there is no explicit incentive to coordinate on the Pareto-dominant equilibrium. Another important difference is that the dying seminar is a binary choice game, whereas order-statistics games offer more than two possible strategic choices.

\section{Experimental design}

In our experiment $N$ subjects are asked to play simultaneously in four different seminars. At the beginning of the experiment each subject is given an individual threshold $H_{i}(1 \leq i \leq N)$, which is in principle different for each seminar. The $H_{i}$ are constant during the game, and are drawn from distributions $f(H)$, which are different for each seminar and correspond to two types of seminar profile, as explained later. In each session the four games are played by the same subjects for $T$ periods. At each period, and for each seminar, each subject gets an initial endowment $W$ that he earns whenever he decides not to attend the seminar. If subject $i$ participates at period $t$ and the actual number of participants $P(t)$ is not less than his threshold $H_{i}$, he keeps the endowment and gets a supplementary payoff, such that his earning is $U$. If $P(t)$ is less than his threshold he loses his endowment, so that in no case the payoffs are negative; referring to equation (1), this means $V=0$. A subject's earnings for all $T$ periods are cumulated, which in principle should act as an incentive for early convergence to the efficient equilibrium. 


\subsection{Characteristics of the four seminars}

The thresholds' distributions $N f(H)$ of the seminars and their cumulative distributions $N F(H)$ are plotted in Fig. 1. The figures on the left correspond to distributions $f(H)$ with a central maximum and two wings, like a $\mathbf{W}$, so we call them W seminars (SW1 and SW2). On the right side are displayed the distribution functions of the $\mathbf{M}$ seminars (SM1 and SM2) where the shape of the $f(H)$ distribution looks like an M. These shapes are important because they determine the types of Nash equilibria of the games, which are given by the intersections of the underlying cumulative functions with the diagonals, as explained in section 2. Fig. 1 shows that seminars SM1 and SM2 have stable equilibria with intermediate attendance. In contrast, seminars SW1 and SW2 show equilibria with either full (or almost full) attendance, or with a very small one; the latter case represents the death of the seminar.

All four seminars have a unique Pareto-optimal Nash equilibrium with full attendance, i.e., perfect coordination of the $N$ individuals ${ }^{2}$. Besides the usual stable and unstable equilibria, seminars SW1, SM1 and SM2 present pure coordination problems within sub-populations that share the same threshold value. These situations exist whenever the line $y=H$ does not intersect the cumulative distribution $N F(H)$, as in SW1 at $H=8$. An attendance of $P=8$ individuals would require participation of only two of the three subjects having a threshold $H=8$. If at least two of these individuals decided to participate, then the third one would not run any risk in participating, thus allowing the system to overcome the critical mass and reach the $P=14$ Nash equilibrium. Otherwise (i.e. if only one of the individuals with $H=8$ attended) the whole system might not reach the higher attendance equilibrium. Such a situation, where the critical mass is not an (unstable) equilibrium, cannot arise in infinite populations with smooth threshold distributions, as explained in section 2 .

In addition to these features, each seminar presents the following characteristics:

SW1 has three stable Nash equilibria, at $P=3, P=14$, and $P=16$. Notice that $P=0$ is not an equilibrium because individuals with thresholds $H=0$ and $H=1$ have a strictly dominant and non-strategic choice: $\omega=1$. The critical mass, $P=8$, is not an equilibrium.

SW2 has two stable Nash equilibria at $P=2$ and $P=16$. Notice that $P=15$ is an unstable equilibrium: if there are already $P=15$ participants, the individual with $H=16$ can improve his payoff through participation without altering the others' payoffs. The critical masses are $P=8$ and $P=15 . P=1$ is not a critical mass: decisions of individuals with $H=1$ are non-strategic, and their participation drives the system to the Nash equilibrium with $P=2$.

SM1 has three stable Nash equilibria, at $P=0, P=7$ and $P=16$. None of the critical masses ( $P=2$ allowing the system to reach the equilibrium $P=7$, and $P=14$ allowing the

\footnotetext{
${ }^{2}$ If some individuals had a threshold larger than $N$, then full attendance would not be a Nash equilibrium. However, those individuals would not have the opportunity of getting the highest payoff in the experiments, because having $H_{i}>N$ ensures that the single winning strategy is to never participate in the seminar. Giving individuals such thresholds would have been unfair, since they would penalize them. This is why we restricted distributions to $0 \leq H_{i} \leq N$.
} 

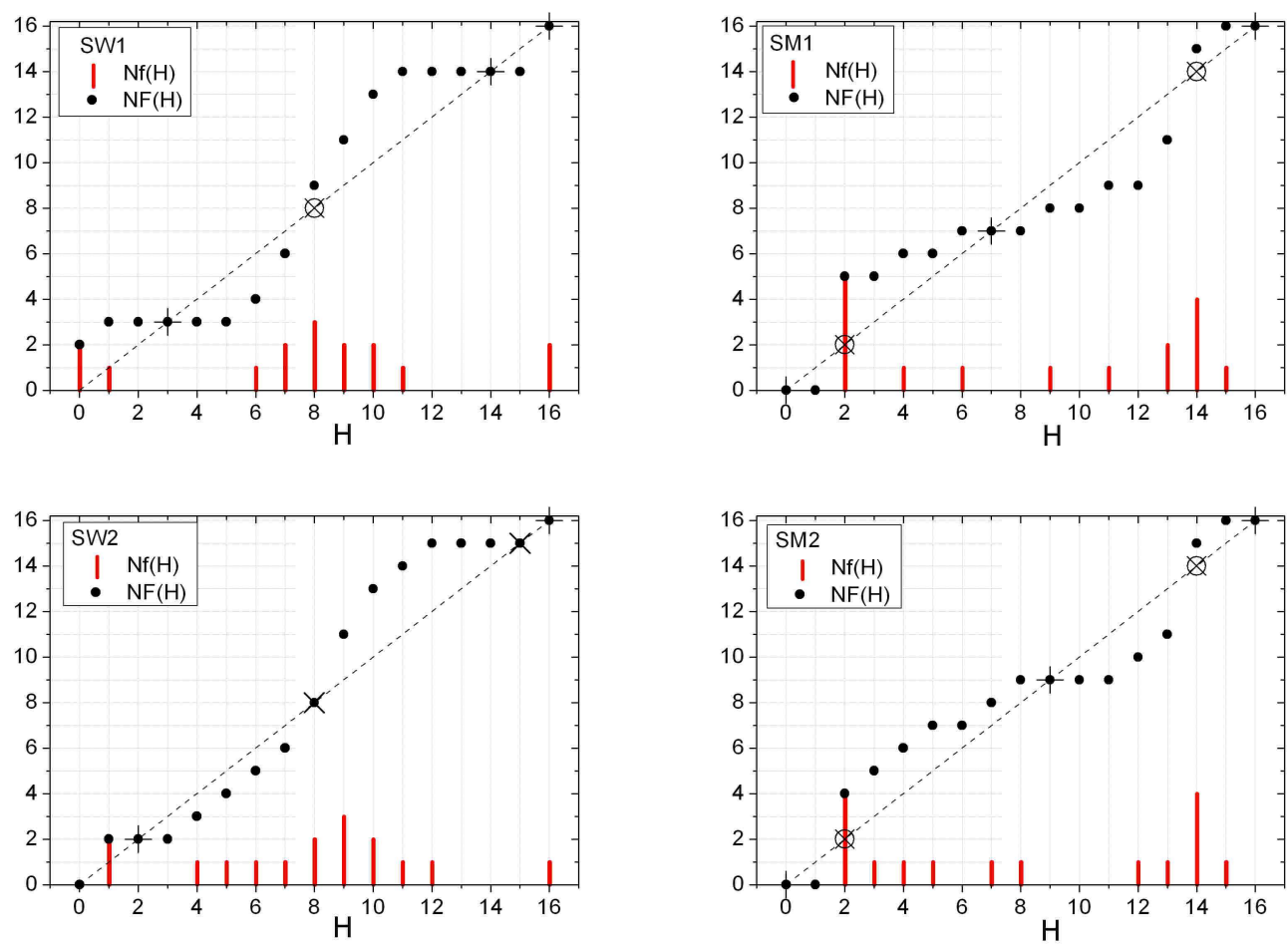

Figure 1: Stable (Nash) equilibria $(+)$ of the experimental seminars. Critical masses that are unstable equilibria $(x)$ and those that are not equilibria $(\otimes)$ are also represented.

system to reach full attendance) are (unstable) equilibria.

SM2 has three stable Nash equilibria, at $P=0, P=9$, and $P=16$. Also in this case the critical masses $P=2$ and $P=14$ are not equilibria.

Seminars SM1 and SM2 are particularly interesting because they require risk-prone subjects in order to reach large attendances. This may be explained if we assume a sequential myopic dynamics, in which individuals decide to participate one after another based on their thresholds - which is the dynamics underlying Schelling's construction - For example, in seminar SM1, individuals with thresholds 9, 11 and 13 need to make risky choices in order to drive the system to an equilibrium attendance larger than 7 . More generally, this arises whenever $N F(H)$ has a slope smaller than 1 at the critical mass, as in the M shaped seminars SM1 and SM2.

\subsection{The four treatments}

In the experiment the subjects know their own thresholds and the payoffs they earned in all previously played periods. We considered four treatments, defined by the following supplementary 
Table 1: The four treatments: the amount of information decreases from top to bottom.

\begin{tabular}{|c||c|c|}
\hline Treatments & A priori & Behavioral \\
\hline \hline OL & yes & P (on-line) and payoffs \\
\hline NP & no & P at all preceding periods and payoffs \\
\hline H & no & whether $H_{i}$ reached at period $t-1$ and payoffs \\
\hline E & no & only payoffs \\
\hline
\end{tabular}

information provided to the subjects:

On Line (OL). Each subject is given the values of all the other subjects' thresholds. Also, within each period, the on-line (i. e. without waiting until the end of the period) decisions of all the individuals, as well as their decisions on all preceding periods are displayed. Thus, when making his decision, each subject knows the number of individuals that already decided to participate, as well as their threshold values. This treatment provides a priori maximal chances for an efficient coordination.

Number of Participants (NP). Each subject is given the number of participants in each seminar at all preceding periods, but this information is refreshed after each period. Thus, each subject knows the past attendances and his own payoffs, but not the other subjects' thresholds nor their decisions at the time he must make his own decision.

Thresholds (H). Each subject, independently of his decision, is informed whether the number of participants in the last period reached his threshold.

Earnings (E). Only the subject's payoffs of the preceding period and the cumulated gains are displayed. Each subject can deduce from these payoffs whether his threshold was reached or not: if he did not participate in a given period, the payoff (which is the endowment) does not carry any information about the period's outcome. The only way subjects can obtain any information is through participation, with the risk of loosing the endowment.

Thus, we experiment with a priori and behavioral information. The information given in the different treatments, summarized in Table 1, decreases gradually from the top to the bottom of the Table. A priori information - i.e. the distribution of the idiosyncratic thresholds - is provided only in treatment OL, giving the subjects the opportunity to determine the equilibria of each seminar from the very beginning. In all the treatments we provide behavioral information and always the individual earnings. OL can be taken as the control treatment, and will serve as base-line reference for the others. In both NP and $\mathrm{H}$ subjects know whether the prior decisions were correct, but in $\mathrm{H}$ they do not know how much larger the attendance was than their individual threshold. In treatments $\mathrm{H}$ and $\mathrm{E}$ subjects get the same information if they participate; however, if they do not participate, the information provided in treatment $\mathrm{E}$ is minimal. 


\subsection{Procedure}

A total of 11 computer-controlled experimental sessions were conducted at the INPG (Institut National Polytechnique de Grenoble); a first set between January and April 2006 (4 sessions), and two additional sets in October 2006 (3 sessions) and April 2008 (4 sessions). In the following section we describe the earlier results. The second set of tests confirmed the earlier findings, and are described in section 5.3. In each session $N=16$ subjects had to decide at each of $T=15$ periods whether to attend each of four seminars. The different information treatments were implemented in separate experimental sessions, with different subjects, to guarantee the independence of the results. Subjects were graduate and undergraduate students who responded to an e-mail sent to different university departments in Grenoble. Some of them had some knowledge of game theory, acquired in their classes.

Upon arriving at the laboratory, the subjects were randomly seated in front of computer terminals. Communication between subjects was strictly forbidden. The instructions were read aloud before the experiment started of, to ensure that all subjects understand the rules, and that they are common knowledge. Paper copies of these instructions were distributed among the subjects. Before each period each subject was endowed with $W=50$ yens (converted to euros at the end of the experiment at the rate 100 yens $=1.45$ euro). The subjects were told that the yens gained or lost during the experiment would be added or subtracted from their initial endowment. After they completed reading the instructions, the subjects played T periods. In our setting, $U=200$ yens and $V=0$ yens (see eq. (1)). Sessions lasted about 1 h 30 . At the end of the session the subjects were paid an amount equivalent to their cumulated yens in one of the four seminars, which was selected at random, and was the same one for all subjects. The fact that only one randomly selected seminar would actually be remunerated was announced in the instructions, so that the subjects played all seminars with the same interest, knowing that they would not be able to compensate losses in one seminar with gains in another.

In each session, information concerning each of the four seminars was displayed on four squares on the subject's screen. His personal threshold for each seminar was indicated in this square. The subject could make his decisions for the current period $t(1 \leq t \leq T)$ in any order: he only needed to mouse-click the chosen seminar, enter his choice and validate it. Once all the subjects had validated their decisions for all four seminars on their terminals, the period was closed and the subject's gain of the period was displayed on the screen in each seminar's square together with the treatment-dependent information. Then, a new period begins.

At the end of each session, subjects were asked to answer anonymously the following questions on paper: (1) which criteria did you use to make your first period decision; (2) what strategy did you follow to make decisions in subsequent periods; (3) did you use information about your past earnings; (4) do you know what game you have been playing. The answers were used to enrich the interpretation of the experimental results, and to identify the information that most affected the subjects' decisions. Subjects were paid after the completed questionnaires were returned to the experimenter.

If coordination were achieved from the start, repeated play of the payoff-dominant equilibrium at all periods of a seminar would result in a subject's earning of 3000 yens (43.50 euro). 
This sets the upper bound to the individual payoffs for this game. Conversely, if the seminar dies from the start, i.e. from the very first period subjects never participate, each subject earns the endowment, obtaining 750 yens (10.875 euro). A subject making completely irrational decisions may earn 0 yens.

\section{$5 \quad$ Results}
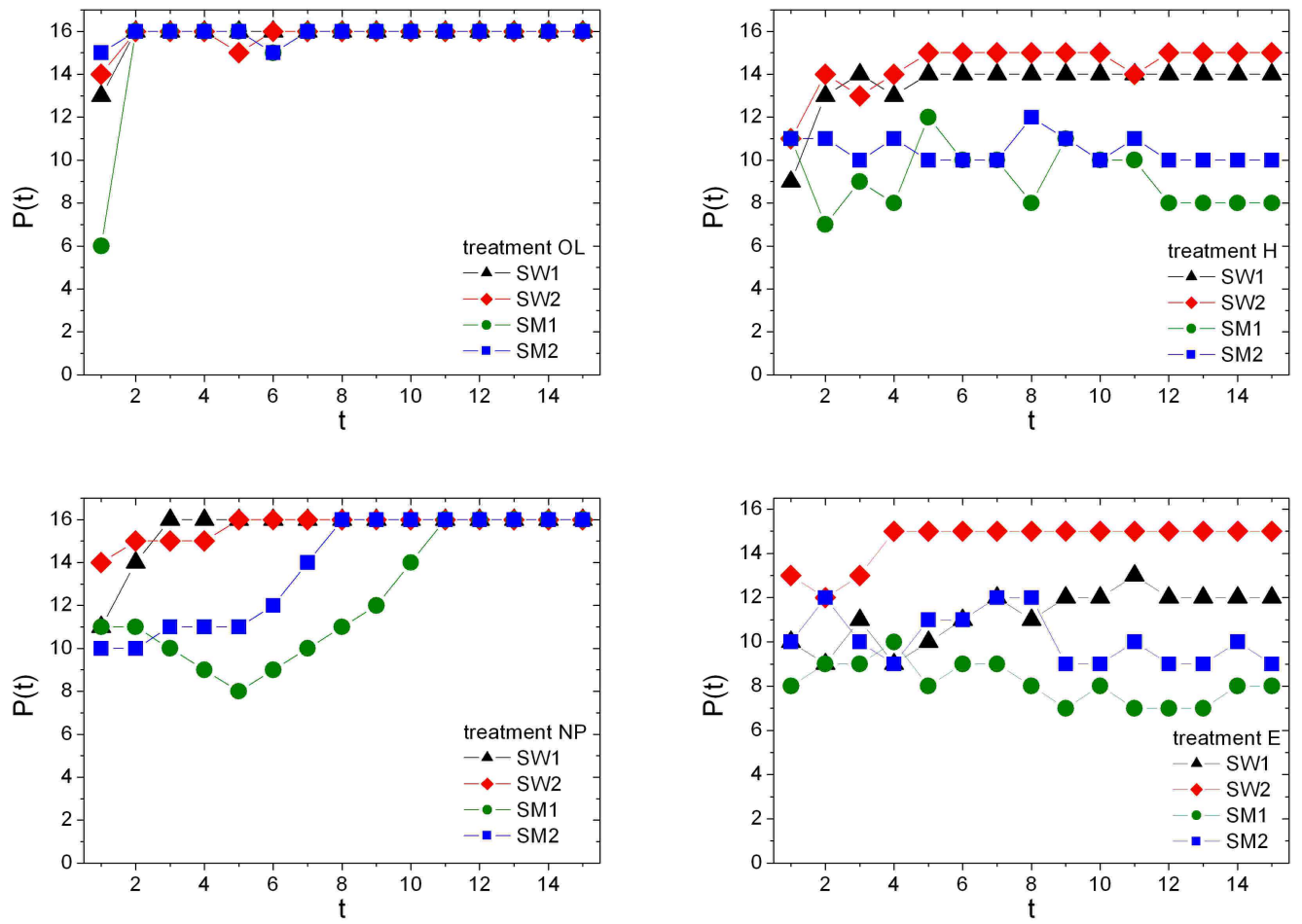

Figure 2: Aggregate results: number of participants in the four seminars as a function of the period, for each treatment.

In this section we discuss the adaptive behavior of the subjects along the successive periods for each of the four treatments. The aggregate results, the number of participants as a function of the period, are represented in Fig. 2. In particular, we analyze the first-period decisions under the OL treatment, because we know in what order the subjects' decisions were made.

\subsection{Treatment OL}

With treatment OL, the critical masses that allow the system to reach the Pareto-dominant equilibria were reached from the first period in all the seminars except SM1. The Pareto-optimal 
equilibrium with complete attendance (16 participants) was achieved in all the seminars as early as the second period. After comparison with the other treatments detailed below we found, not surprisingly, that providing complete information speeds up convergence dramatically. These results agree with experimental economics literature: full information represents a potentially powerful coordination mechanism, as subjects may decide to participate at the time when their thresholds are reached, or may participate through "imitation" of others (who may or may not share their thresholds). The drop in attendance in the middle of the experiment in seminars SW2, SM1 and SM2 are due to subjects who unsuccessfully tried to modify the collective dynamics, as can be deduced from the answers to the questionnaire.
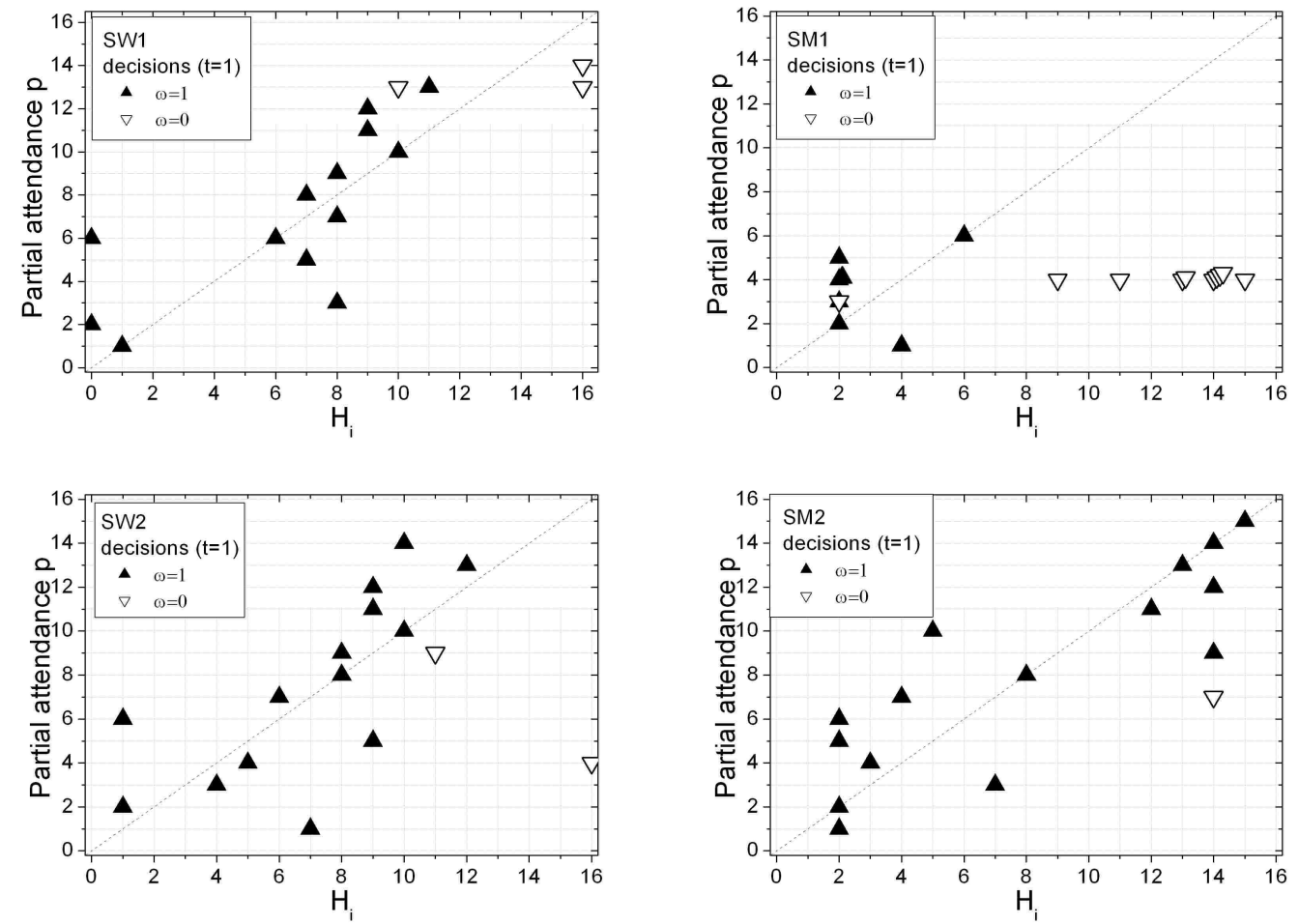

Figure 3: On Line treatment: partial attendance $p$ as a function of the thresholds: the attendance immediately after the subject with threshold $H_{i}$ has made his decision. Here, black triangles are decisions $\omega=1$, and empty triangles are decisions $\omega=0$. e.g. in seminar SW1, the subject with $H=8$ chooses to attend when $p=2$ (after subjects with $H=1$ and $H=0$ have participated). Decisions of the subjects sharing the same thresholds are shown as overlapping (seminar SM1).

First-period decisions. The treatment OL is especially adapted to understanding the behavior of subjects in the first period, since we can correlate the order of the decisions with the subjects' thresholds. In Fig. 3 we plot the partial attendance $p$ - the number of participants 
immediately after a subject with threshold $H_{i}$ has made his decision-, as a function of the (ordered) thresholds. Decisions to attend $(\omega=1)$ or not $(\omega=0)$ are represented with different symbols. In these figures, time increases along the y axis: the very first decision takes place when the partial attendance is $p=0$, the next one when $p=1$, and so on. Decisions to attend $(\omega=1)$ lying along the diagonal $y=H$ are evidence of strictly rational behavior, since these subjects decide to participate exactly when their thresholds $H_{i}$ are reached. Decisions $\omega=1$ lying above the diagonal point to winners, since attendance is already larger than their thresholds. Conversely, subjects whose decision to participate lies below $y=H$ take a risk, since in this case attendance $p$ is less than their thresholds. For example in seminar SW1, one of the two subjects with $H=7$ who decides to participate when $p=4$, and two of the three subjects with $H=8$, who decide to participate when $p=2$ and $p=6$ respectively, show a risk-prone behavior. This is even more conspicuous in seminar SW2, where the subject with $H=7$ is the first one that decides to attend. He may benefit from this decision only if at least six other subjects participate, as was indeed the case in the experiment.

Decisions not to participate that lie above the diagonal, like that of the subject with $H_{i}=10$ in seminar SW1, whose $\omega_{i}=0$ when $P=12$, are not rational.

In seminar SM1, despite the risky behavior of the subjects with $H=4$ who were the first that decided to attend, the non-rational behavior of one of the subjects with $H=2$, who decided not to attend, hindered coordination on the lowest Nash equilibrium with $P=7$. On the other hand, in seminar SM2 the risky behavior of many subjects (one with $H=2$, one with $H=7$, one with $H=12$ and two with $H=14$ ) drive the system to a high attendance. The early risk-averse decision not to attend of the subject with $H=14$ hindered convergence to the Pareto optimum.

Clearly, the behavior that we call risky may actually be teaching strategies: these subjects may be trying to induce others to attend. However, we do not have evidence that this was actually their aim.

Because the distribution $f(H)$ in treatment OL is of common knowledge, we can analyze the pure coordination problems that face sub-groups of subjects sharing the same threshold value (see section 4.1$)^{3}$. Coordination failures of these subjects may hinder convergence to the Nash equilibrium. In seminar SW1 the subjects with $H=10$, who did not coordinate during the first period, are responsible for attendance $P(0)=13$ (less than the Nash equilibrium $P=14$ ). In seminar SM1 all the subjects with $H=14$ appear to be risk-averse, while in seminar SM2, because of the risk-averse decision of one of the subjects, the subgroup with $H=14$ failed to coordinate, leading to attendance $P=15$. Notice that the subjects with $H=14$ that are risk-averse in seminar SM1 are not the same ones that have these thresholds in seminar SM2.

Treatment NP. As represented in Fig. 2 (lower left), coordination on the Pareto-optimum is reached in all seminars, but requires more time than under the reference treatment OL. Knowing the period-by-period attendance may induce a myopic-like learning from one period to the next: if the number of participants is less than the subject's threshold he refrains from participating;

\footnotetext{
${ }^{3}$ This study was carried out on data of the first period, the only one in which choices are strategic since full attendance was already reached at the second period.
} 
otherwise he participates. This behavior was observed in all four seminars, though not in all subjects.

Interestingly, there is a clear correlation between the seminars' attendances: they converge one after the other. After saturation of seminar SW1, seminars SW2 and SM2 begin to increase their stagnant attendance to converge one after the other. This effect, indicating that subjects learn to coordinate based on the success of other seminars, is even more conspicuous in seminar SM1, which changes from a decreasing to an increasing attendance dynamics immediately after convergence of seminar SW2.

Treatment H. Since subjects do not know how far the actual attendance $P(t)$ is from their threshold, participation entails a risk for all non-participants, independently of their threshold. Despite the scarce (private) information we still observe good coordination (see Fig. 2, upperright), though not enough to reach the Pareto-optimal equilibrium. Subjects with $H_{i}=P(t)+1$, who might improve their earnings through participation if they knew the actual attendance, may not risk participating. This explains the relatively slow convergence of seminars SM1 and SM2, that reach their equilibria only after 12 periods.

Treatment E. Information available is strictly private: subjects know only their own actions and earnings. Only those subjects that take the risk of participating may obtain information about the attendance level. The global behavior is different from that emerging from the other treatments (see Fig. 2, lower-right). There are more temporal fluctuations in the aggregate attendance. The attendances of seminars SM1 and SM2 do not stabilize until after 15 periods, mainly because of the non-rational behaviors of some subjects, as will be explained below. However, despite the low information level, a good coordination level is observed, and none of the seminars dies. Attendance in seminar SW1 reached $P=12$, lower than the Nash equilibrium $(P=14)$. The subject with $H=0$ never participated, and he stated in the questionnaire that he did not understand the meaning of a zero threshold. The subject with threshold $H=9$ chose not to participate from the start and did not try to learn. Otherwise the system might have reached the stable Nash equilibrium with $P=14$. In seminar SW2, after two attempts with unsuccessful results, the subject with $H=16$ stopped participating. This is why the attendance at convergence was $P=15$. Besides these particular behavior, it would seem that the willingness to get large payoffs overcomes individual risk-aversion. This may also be a consequence of the small size of the group: as in the stag hunt experiments by [Van Huyck et al.(1990)], we expect coordination of larger groups to become increasingly difficult.

\subsection{Seminar types}

We aggregated the results of the four different treatments for each seminar, in Fig. 4. Depending on the shape of the underlying thresholds mass distribution, (W-or M-shaped see discussion in section 4), there appears to be clear differences in the type of collective dynamics.

Seminars SW1 and SW2 (see Fig. 4, left), with a central peak in the thresholds mass distribution, present a remarkable coordination even with treatment $\mathrm{H}$, in which the individual 

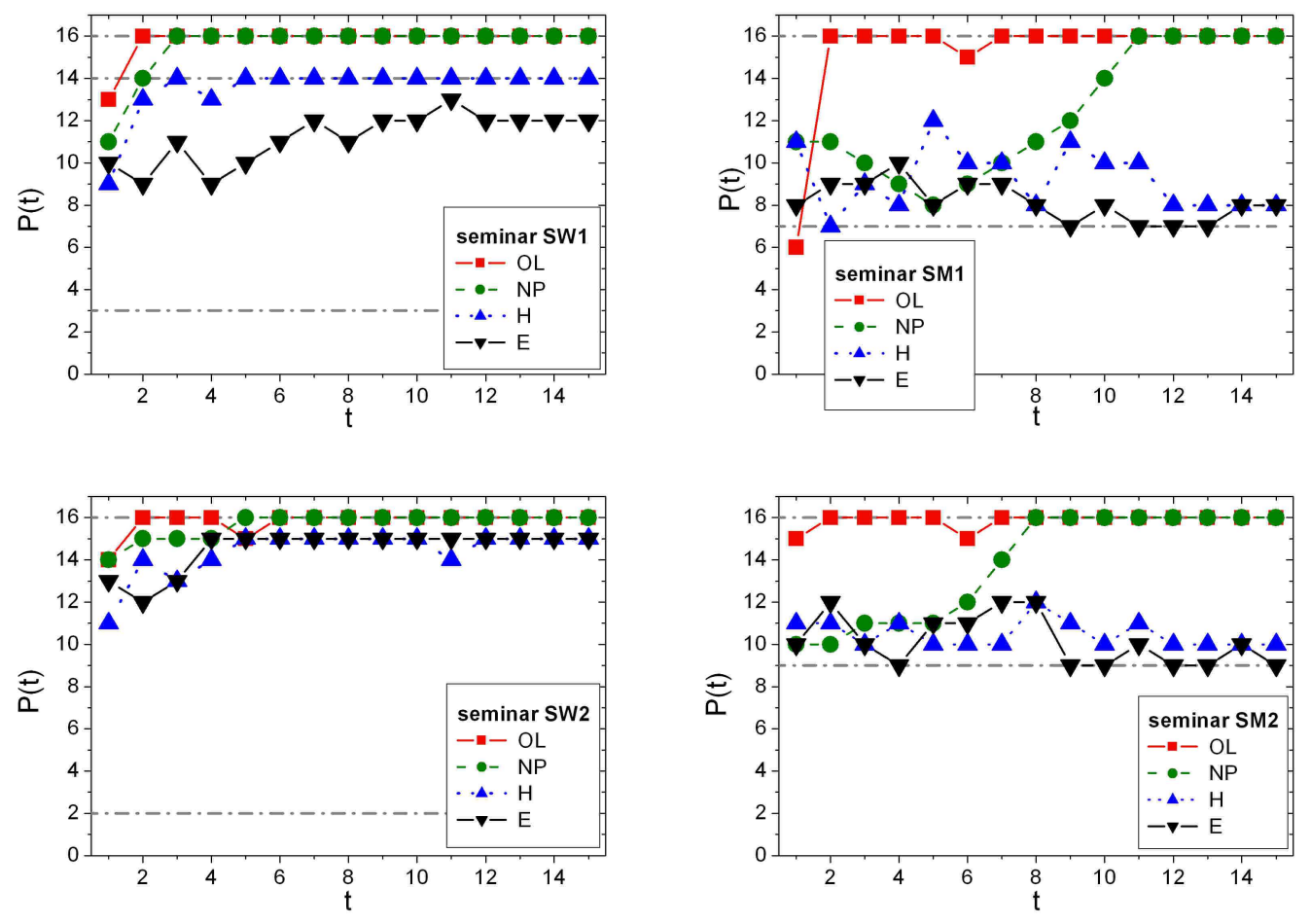

Figure 4: Aggregate results by seminar type: number of participants as a function of the period for each seminar separately, and for four treatments within each seminar. Nash equilibria are represented as dash-dot horizontal lines.

risk is higher because subjects do not know the exact attendance at each period, in contrast with the results by [Devetag(2003)].

In treatment $\mathrm{H}$, seminars SM1 and SM2 reach intermediate attendance, that fluctuates close to the stable Nash equilibria. Interestingly, these fluctuations are systematically positive: the attendances are equal or larger than those expected at the Nash equilibrium. In order to get a deeper insight on the subjects' dynamics, we present in Table 2 the payoffs obtained by all subjects at each period. The first row lists the subjects' identifiers, ordered by their thresholds, listed in the second row; in the last column we report the number of participants at each period. Intersections of each column and row give the individual payoff for the period. In seminar SM1 subject 6 with threshold $H=9$ fixed a priori his strategy to participate and never changed it, without taking into account his frequent loss of the initial endowment. In seminar SM2 subjects with $H=15$ and $H=14$ insisted in participating in subsequent periods despite their repeated losses. Clearly, subjects whose thresholds are lower than the Nash equilibrium attendance behaved rationally: they all attended after a few periods (see Table 2, to the left of the heavy vertical line). Fluctuations in decisions result from risky attempts by individuals with 


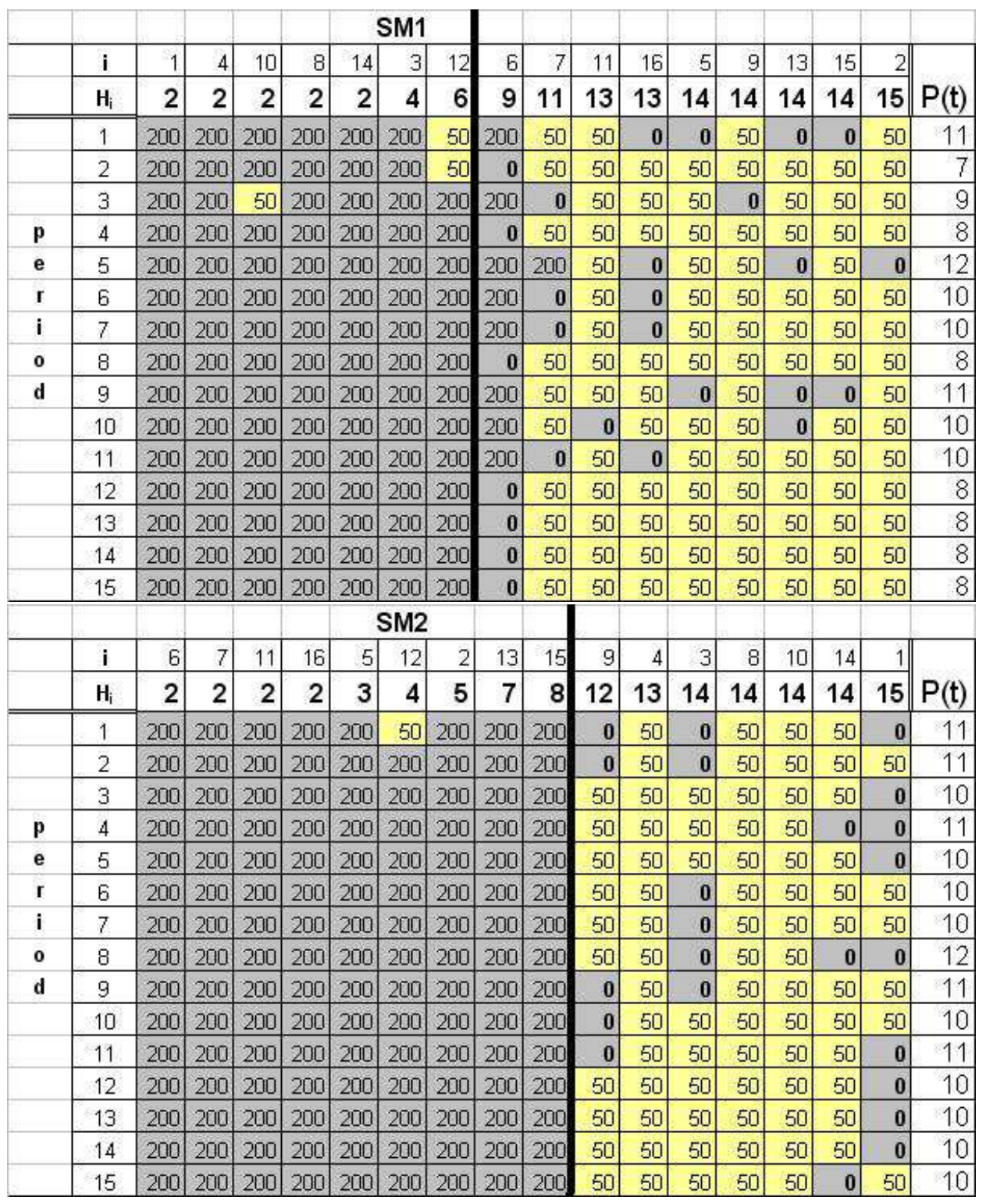

Table 2: H treatment: Payoff matrices for seminars SM1 and SM2. The color of the cells indicates the chosen strategy: grey for participation, yellow for non-participation. The numerical values inside each cell are the subject's payoffs earned for the period. Heavy black vertical line indicates the Nash equilibrium.

higher thresholds, that may be interpreted as unsuccessful attempts to induce full attendance by teaching. These fluctuations are more conspicuous in treatment E (see Fig. 4), in which the only way to find out the actual attendance is to take the risk of participating. 


\subsection{Summary}
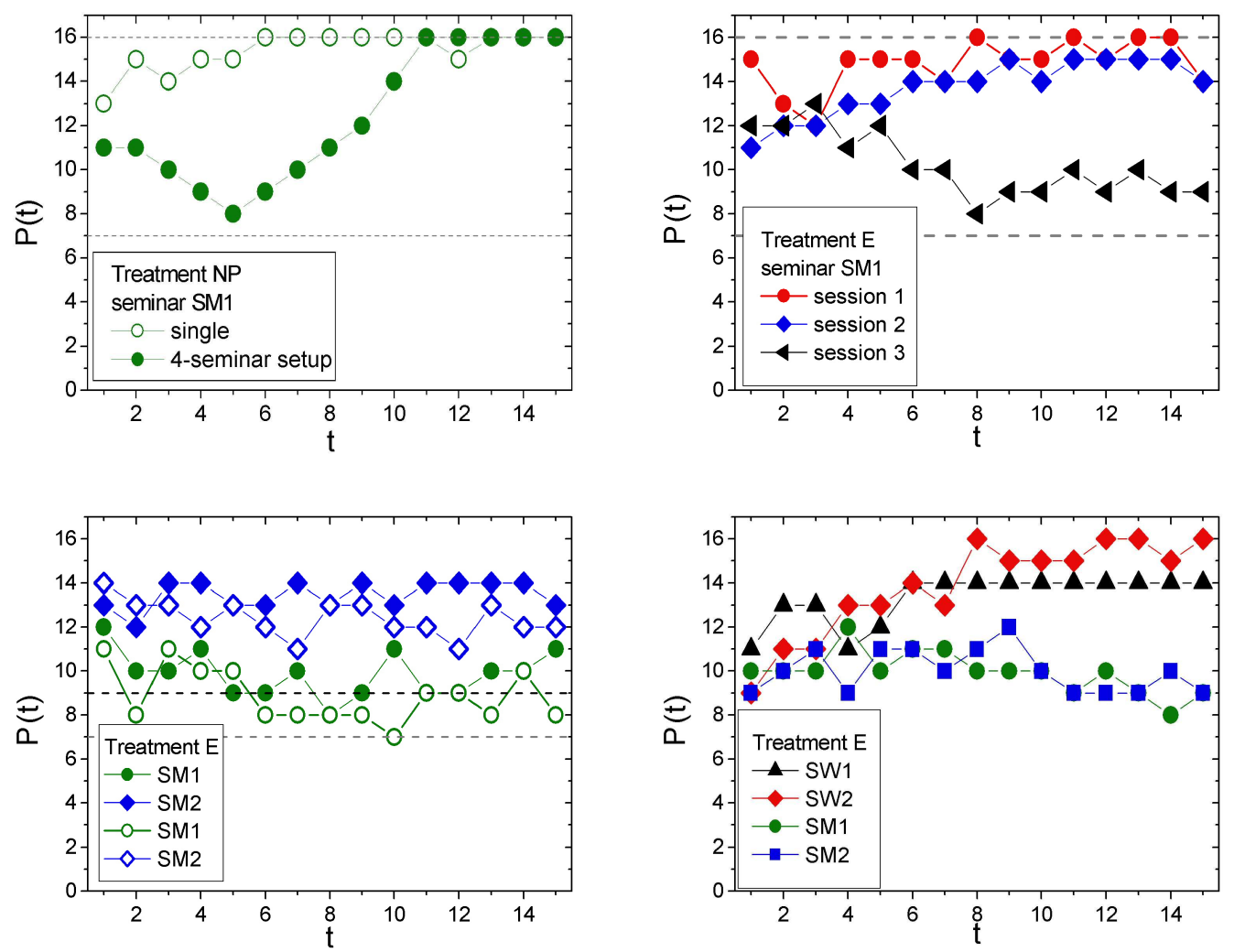

Figure 5: Aggregate results: number of participants as a function of the period, for additional sets of experimental sessions. Upper-left: seminar SM1 played alone under treatment NP; Upper-right: seminar SM1 played alone in three sessions under treatment E; Lower-left: seminars SM1 and SM2 played simultaneously in two sessions under treatment E; Lower-right: four seminars played simultaneously under treatment E. Nash equilibria are represented as dash-dot horizontal lines.

A short summary of the experimental results is presented in Table 3. The principal observation is that even in the absence of complete information an efficient coordination is possible. This efficiency depends strongly on the information available and on the pattern of idiosyncratic preferences. Less information results in longer convergence times. Even when the information available is strictly private attendance reaches at least a sub-optimal equilibrium.

An unexpected result of our experimental setup (see seminar SM1 under treatment NP in Fig. 2 ) is that convergence to equilibria with perfect coordination may be induced by the simultaneous playing of other, less risky, games. However, we verified that this spillover effect does not explain the large participation levels obtained experimentally even under lower information conditions. 
To this end, we carried out several independent sessions. The setups and main conclusions are summarized as follows:

1. Equilibrium attendances $P \geq 14$ were reached in three sessions where SM1 played alone, once under treatment NP (see Fig. 5, upper-left) and twice under treatment E (see Fig. 5, upper-right, sessions 1 and 2). Moreover, with treatment NP perfect coordination was reached after period 6, i.e. much earlier than in the four-seminar setup, indicating that convergence to the Pareto-optimal equilibrium does not require the spillover effect. In these sessions, fluctuations in the number of participants from period to period are larger than in the four-seminar setup.

2. Seminar SM1 reaches attendances fluctuating between 8 and 10 when played alone under treatment E (see Fig. 5, upper-right, session 3). Perfect coordination was not reached, but the attendance level presents the same trends as in the four-seminar setup. The game was played for 25 periods, to determine whether the fluctuations were due to early stopping, an indication that fluctuations are inherent to the small amount of information available.

3. We conducted two sessions with two seminars played together, SM1 and SM2, under treatment E (see Fig. 5, lower-left). The results reproduce the qualitative trends of those in the four-seminar setup.

4. Finally, we made one more session with the four seminars played simultaneously under treatment E (see Fig. 5, lower-right). The results present the same trends as in the earlier sessions. Again, the W-type seminars converge to larger attendances than the M-type ones.

Table 3: Summary: Pareto-ranking and periods for convergence. Abbreviations: PO: Pareto optimal, PD: Pareto dominant, SE: stable equilibrium (theoretical). The numerical values in parenthesis are the periods required for convergence.

\begin{tabular}{|c|c|c|c|c|}
\hline & Treatment OL & Treatment NP & Treatment H & Treatment E \\
\hline SW1 & PO $(2)$ & PO $(3)$ & PD $(5)$ & $<\mathrm{PD}(12)$ \\
\hline SW2 & PO $(2)$ & PO $(5)$ & PD $(5)$ & PD $(4)$ \\
\hline SM1 & PO $(2)$ & PO $(11)$ & $\gtrsim S E(12)$ & $\gtrsim \mathrm{SE}(>15)$ \\
\hline SM2 & PO $(2)$ & PO $(8)$ & $\gtrsim S E(12)$ & $\approx \mathrm{SE}(>15)$ \\
\hline
\end{tabular}

\section{Learning models}

In this section we present our attempts to interpret the results of our experiment in terms of agents' learning. We simulate a system of $N=16$ agents that periodically decide whether or not to participate under the four treatments: OL, NP, H and E, presented in section 4.2. 


\subsection{Learning dynamics}

Following [Camerer(2003)], we assume that agents associate attractions to each strategy. These attractions determine the probabilities with which strategies are chosen by the agents when playing the game repeatedly. In problems of binary decisions, it is sufficient to consider the attraction for one strategy, say participating, with respect to the other: $A_{i}(t)$. Depending on the informational setting, these attractions may be modeled by the difference between the agent's expected number of participants $\hat{P}_{i}$ and his threshold $H_{i}: A_{i}(t)=\hat{P}_{i}(t)-H_{i}$, or by the expected payoff upon participating, which in our experiments can take either of two values: $A_{i}=U-W$ or $A_{i}=V-W$.

Before making his first decision, each agent $i$ starts with an attraction $A_{i}(0)$ that reflects his initial belief. Agents may begin with totally random estimations or with estimations correlated with their thresholds. Then, at each period $t$ each agent $i$ chooses $\omega_{i}(t)$ following a decision rule that depends on the attractions $A_{i}(t)$. After their decisions are made, agents receive the corresponding payoffs and the supplementary information corresponding to the treatment considered. Then, attractions are updated using a learning rule based on the information obtained from the previous periods. These two steps are explained hereafter.

Decisions. Since experimental results show that subjects not always choose optimal actions at every period, we assume a trembling hand dynamics ${ }^{4}[\operatorname{Selten}(1975)]$ so that both strategies have positive probabilities of being selected. If an agent's best response is

$$
\omega_{i}^{B R}(t)=\Theta\left(A_{i}(t)\right)
$$

(where $\Theta$ is the Heaviside function, i.e. $\omega_{i}^{B R}(t)=1$ if $A_{i}(t) \geq 0$ and $\omega_{i}^{B R}(t)=0$ otherwise), then at each period $t$ each agent $i$ chooses a strategy $\omega_{i}(t)$ according to

$$
\begin{array}{ll}
\omega_{i}(t)=\omega_{i}^{B R}(t) & \text { with probability } 1-\epsilon \\
\omega_{i}(t)=1-\omega_{i}^{B R}(t) & \text { with probability } \epsilon
\end{array}
$$

where $\epsilon$ is a small positive number $0<\epsilon<1$, for simplicity assumed to be the same for all $i$.

Updating attractions. According with the subjects' responses in the questionnaire ${ }^{5}$, we assume that agents are myopic: they respond only to the information obtained in the previous period.

In treatments $\mathrm{OL}$ and NP, agent $i$, independently of whether he participated or not in the previous period, knows the actual number of participants $P(t)$. His attraction is updated according to:

$$
A_{i}(t+1)=P(t)-H_{i}
$$

\footnotetext{
${ }^{4}$ We have simulated a strictly best reply dynamics, and the results obtained do not fit the empirical ones.

${ }^{5}$ Subjects responded that they used only the information of the preceding period, disregarding older informations.
} 
In treatment $\mathrm{H}$ agents that attended the seminar use the differences between the earned payoffs and the endowments to update attractions. Those that decided not to attend know only whether their threshold has been reached or not. The attractions are thus updated as follows:

$$
A_{i}(t+1)= \begin{cases}\pi_{i}(t)-W & \text { if } \omega_{i}(t)=1 \\ 0 & \text { if } \omega_{i}(t)=0 \text { and } P(t) \geq H_{i} \\ A_{i}(t) & \text { if } \omega_{i}(t)=0 \text { and } P<H_{i}\end{cases}
$$

where $\pi_{i}$ is given by (1).

In treatment $\mathrm{E}$, individuals that did not attend the seminar do not get any information, and we assume that in this case they keep their attraction values unmodified. Thus,

$$
A_{i}(t+1)= \begin{cases}\pi_{i}(t)-W & \text { if } \omega_{i}(t)=1 \\ A_{i}(t) & \text { if } \omega_{i}(t)=0\end{cases}
$$

\subsection{Simulations results}

In Fig. 6 we present the results of our simulations. The number of participants as a function of the period fits the empirical data well. In all the simulations we used the experimental first period choices as initial conditions. Treatment OL was simulated as a sequential best response dynamics: agents choose their strategies in the same order as the experimental rank of the first period decisions. The agreement with experiments is expected since the system converged at the second time step (see Fig. 6, upper-left).

We simulated the other three treatments using parallel dynamics: at each period the agents make decisions simultaneously. In order to reproduce the correlations observed experimentally, we first sorted the seminars on their convergence times. We started simulating the learning dynamics with $\epsilon>0$ for all seminars. The value of $\epsilon$ (the probability of an irrational decision) is then set to zero for the first seminar that converged experimentally, say after some number of iterations $t_{0}$. When this seminar converges in our simulations, we set $\epsilon=0$ for the second seminar; and so on for the remaining seminars until $T=15$. We use this procedure for all treatments for which the attendance converged. In the case of seminars that did not converge experimentally, instead of putting $\epsilon=0$ (which would necessarily drive the system to a fixed point attractor) we decreased its value to obtain fluctuations of the same order as appear in the experiments. In other words, we manipulate two parameters: $t_{0}$ and $\epsilon$ (the same for all the agents), to reproduce the data as closely as possible. Decreasing $\epsilon$ according to the order of convergence in the experiments allows us to take into account the observed correlation of the attendances of the experimental seminars, that converged one after the other. Notice however that high coordination levels are obtained mainly because the initial attendances are larger than the critical masses, and not due to the spillover effect. Moreover, with lower initial attendances the spillover effect might operate in the opposite direction, driving the systems to small attendance Pareto equilibria.

The simulated results are shown in Fig. 6, and present similar trends as in Fig. 2. We have experimented with many values of $\epsilon$, and the ones that were used in these simulations are indicated inside each legend. 

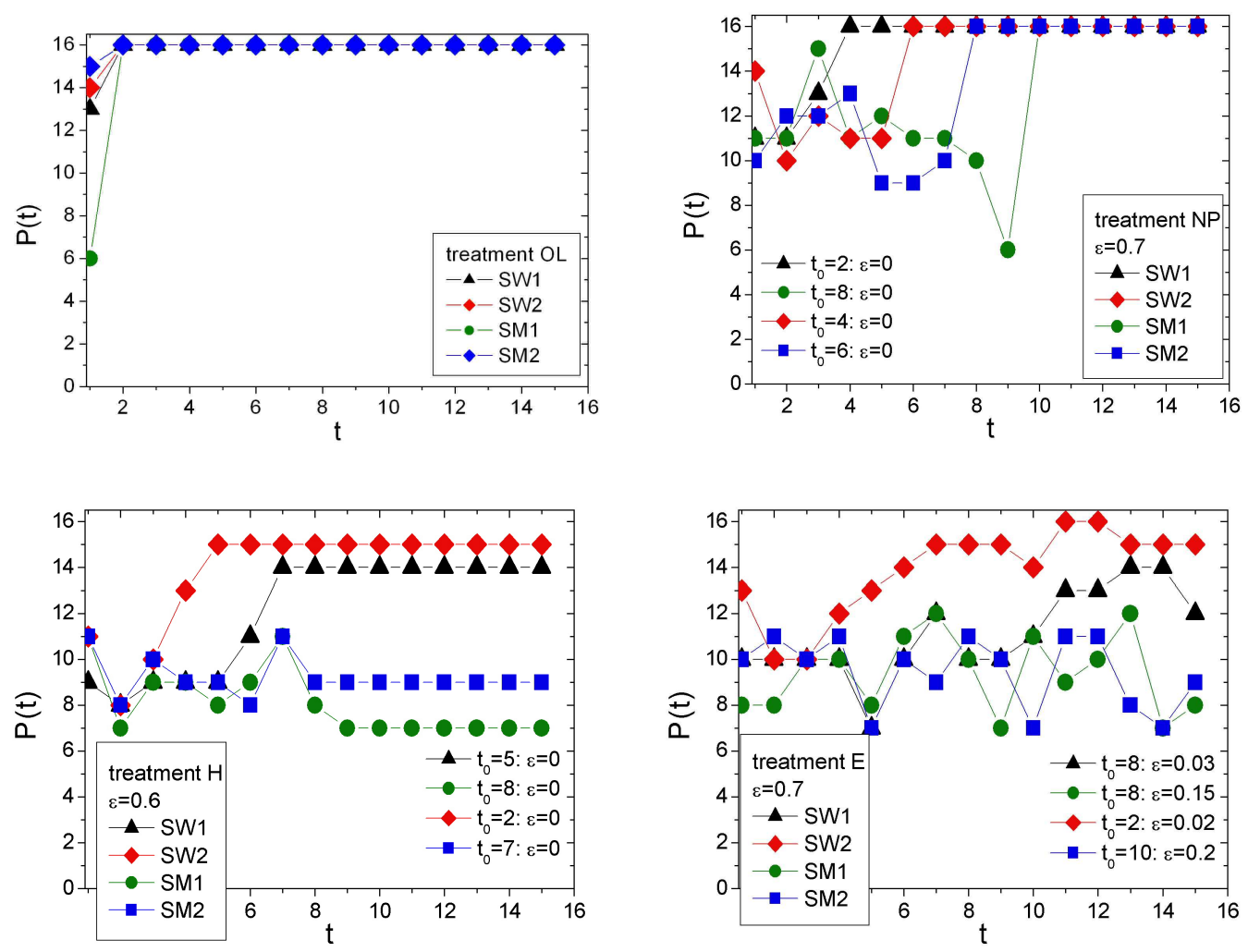

Figure 6: Simulated results with trembling hand decisions: number of participants as a function of the period.

In the case of treatment $\mathrm{E}$, the subject obtain information through their strategic decisions exclusively. This is why we also considered the cumulative proportional reinforcement (CPR) [Laslier et al.(2001)] learning rule, that takes into account the cumulated gains to calculate the probability for each strategy. The fits are slightly better than with the probabilistic myopic learning presented above, probably because, despite their claim, individuals took into account payoffs older than just those of the preceding period.

\section{Concluding remarks}

In this paper we presented experimental results of the dying seminar game, obtained under four treatments, namely $\mathrm{OL}, \mathrm{NP}, \mathrm{H}$ and $\mathrm{E}$ (see section 4.2). The purpose of our experiment was to explore the influence of the information available to the subjects on the game outcome, and to determine the minimum information elements that would allow for an efficient coordination 
among subjects.

The results show that the knowledge of the number of participants, as in treatment NP, is enough information to reach the efficient Pareto-optimal equilibrium. Moreover, even without complete information, sufficient decentralized coordination is possible, though not always on a Pareto-optimum. The level of coordination is mainly limited by the amount of information available and by the pattern of the idiosyncratic preferences, but also by the initial attendance, certainly related to the subjects' risk aversion.

An unexpected result obtained as a result of our experimental setup shows that spillover effects between simultaneously played games may accelerate coordination. Fudenberg et al. [Fudenberg and Levine(1998)] argue that "players do extrapolate across games they see as similar", although they do not consider this question any further in their book. Our results are a step towards the understanding of these phenomena. However, it should be pointed out that the high coordination levels obtained experimentally are not the result of such spillover effects, since they also arise when the games are played separately.

In all the treatments, some subjects chose actions that did not maximize their own payoffs, allowing others to increase their gains. Many researchers accept that individuals are not only self-interested but are also concerned about the payoffs of others. Examples of pure altruism [Fehr and Gachter(2002)] and fairness (where people reciprocate, rewarding kindness and punishing unkindness [Rabin(1993)]) have been reported in the literature. However, the behavior of the subjects in our experiment seem to be different. Selfish behavior has been tested mainly in games where individuals observe the behavior of others. In contrast, in the dying seminar, individuals make decisions simultaneously without knowing the others' choices, so that we cannot deduce whether they are trying to be kind to others. We interpret such behavior as a teaching attitude because, by creating conditions that allow others to gain larger payoffs, they may expect that the attendance in subsequent periods will reach their own thresholds. In other words, these subjects sacrifice gains during some periods, inciting others to participate, and expecting in return to obtain larger payoffs later.

Because of the finite size of the system, we were able to analyze in detail the individual responses to the paper questionnaire, and explain precisely the equilibria reached, as well as the deviations from them. This in turn helps us to determine the factors that influence the subjects' behavior. Clearly, with very large populations it would not be possible to obtain such precise characterizations. In large groups we expect to have the start-up problems described by $[\operatorname{Rohlfs}(2001)]$, of getting large enough initial attendances to reach the critical mass. Thus, there is a possibility that very large groups converge to low attendance equilibria, an outcome that we did not observe in our small groups. Future research involves experiments with higher numbers.

We analyzed the results obtained in terms of the initial states and individual learning. The former may be crucial in determining the dynamical path of the system. In successive periods, as agents gain experience, the important features are the learning behaviors. Our results suggest that individuals take into account more remote experience despite their perception that they only take into account the last outcome. 


\section{Acknowledgements}

We gratefully acknowledge the financial support from the Institut de Production et des Organisations Industrielles (IPI) of Grenoble and from the ACI Complex Systems in Social and Human Sciences (CNRS and French Ministry of Education), project ELICCIR. We thank especially Jean-Loup Dupuis (GAEL, Grenoble) who provided technical assistance by developing the software and helping to conduct the experiments.

MBG is a member of CNRS.

\section{References}

[Artle and Averous(1973)] Artle, R., Averous, C.: (1973), The Telephone System as a Public Good: Static and Dynamic Aspects, The Bell Journal of Economics and Management Science 4(1): 89-100.

[Benabou(1996)] Benabou, R.: (1996), Equity and Efficiency in Human Capital Investment: The Local Connection, The Review of Economic Studies 63(2): 237-264.

[Berninghaus and Ehrhart(2001)] Berninghaus, S. K. and Ehrhart, K-M.: (2001), Coordination and information: recent experimental evidence, Economics Letters 73(3): 345-351.

[Bowles(2004)] Bowles, S.: (2004), Microeconomics: Behavior, Institutions and Evolution. Princeton University Press.

[Camerer(2003)] Camerer., C. F.: (2003), Behavioral Game Theory: Experiments in Strategic Interaction. Princeton University Press, Princeton, New Jersey.

[DeJong et al.(1994)] Cooper, R., DeJong, D., Forsythe, R., Ross, T.: (1994), Alternative Institutions for Resolving Coordination Problems: Experimental Evidence on Forward Induction and Preplay Communication. In: Friedman, J.W. (Ed.). Problems of coordination in economic activity, Kluwer, Boston, Mass, 129-146.

[Cooper et al.(1992)] Cooper, R., Forsythe, R., DeJong, D., Ross, T.: (1992), Communication in Coordination Games, The Quarterly Journal of Economics 107(2):739-771.

[Crawford and Haller(1990)] Crawford, V.P. and Haller, H.: (1990), Learning How to Cooperate: Optimal Play in Repeated Coordination Games, Econometrica 58(3): 571-595.

[Curien et al.(1987)] Curien, N. and Gensollen, M.: (1987), Les théories de la demande de raccordement téléphonique, Revue Economique, 203-255.

[Devetag(2003)] Devetag, G.: (2003), Coordination and Information in Critical Mass Games: An Experimental Study, Experimental Economics 6: 53-73. 
[Devetag and Ortmann(2007)] Devetag, G. and Ortmann, A.: (2007), When and Why? A critical survey on coordination failure in the laboratory, Experimental Economics 10: 331344.

[Durlauf(1996)] Durlauf, S.N.: (1996), A Theory of Persistent Income Inequality, Journal of Economic Growth 1 (1): 75-93.

[Farell(1987)] Farell, J.: (1987), Cheap talk, Coordination and Entry, The Rand Journal of Economics 18(1): 34-39.

[Fehr and Gachter(2002)] Fehr, E. and Gachter, S.: (2002), Altruistic punishment in humans, Nature 415: 137-140.

[Fudenberg and Levine(1998)] Fudenberg D. and Levine D. K.:(1998), The theory of learning in games. The MIT Press, Cambridge,Massachussetts.

[Glaeser et al.(1996)] Glaeser, E.L., Sacerdote, B., Scheinkman, J.A.: (1996), Crime and Social Interactions, The Quarterly Journal of Economics 111(2): 507-548.

[Granovetter(1978)] Granovetter, M.: (1978), Threshold Models of Collective Behavior, The American Journal of Sociology 83(6): 1420-1443.

[Ho et al.(2007)] Ho, T., Camerer, C. F. and Chong, K.: (2007), Self tuning experience weighted attraction learning in games, Journal of Economic Theory 133(1): 177-198.

[Kandori et al.(1993)] Kandori, M., Mailath, G.J., Rob, R.: (1993), Learning, Mutation, and Long-Run Equilibria in Games, Econometrica 61(1): 29-56.

[Katz and Shapiro(1994)] Katz, M. L. and Shapiro, C.: (1994), Systems Competition and Network Effects, The Journal of Economic Perspectives 8(2): 93-115.

[Krauth(2006)] Krauth, B.: (2006), Social Interactions in Small Groups, Canadian Journal of Economics 39(2): 414-433.

[Laslier et al.(2001)] Laslier, J. F., Topol, R., Walliser, B.: (2001), A Behavioral Learning Process in Games, Games and Economic Behavior 37(2): 340-366.

[Nadal et al.(2005)] Nadal, J.-P., Phan, D., Gordon, M. B, Vannimenus, J.: (2005), Multiple equilibria in a monopoly market with heterogeneous agents and externalities, Quantitative Finance 5(6): 557-568.

[Ostrom(2000)] Ostrom, E.: (2000), Collective Action and the Evolution of Social Norms, The Journal of Economic Perspectives 14(3): 137-158.

[Rabenau and Stahl(1974)] Von Rabenau, B. and Stahl, K.: (1974), Dynamic Aspects of Public Goods: A Further Analysis of the Telephone System, The Bell Journal of Economics and Management Science 5(2): 651-669. 
[Rabin(1993)] Rabin, M.: (1993), Incorporating Fairness into Game Theory and Economics, The American Economic Review 83(5): 1281-1302.

[Rankin et al.(2000)] Rankin, F., Van Huyck, J. B., Battalio, R. C.: (2000), Strategic similarity and emergence of conventions: Evidence from payoff perturbed stag hunt games, Games and Economic Behavior 32: 315-337.

[Rohlfs(1974)] Rohlfs, J.: (1974), A Theory of Interdependent Demand for a Communications Service, The Bell Journal of Economics and Management Science 5(1): 16-37.

[Rohlfs(2001)] Rohlfs, J.: (2001), Bandwagon Effects in High-Technology Industries. The MIT Press, Cambridge, Mass.

[Seale et al.(2002)] Seale, D., Rapoport, A., Winter, E.: (2002), Coordination and Learning Behavior in Large Groups with Asymmetric Players, Games and Economic Behavior 39(1): 111-136.

[Selten(1975)] Selten., R.: (1975), Re-examination of the perfectness concept for equilibrium points in extensive games, International Journal of Game Theory 4(1): 25-55.

[Schelling(1978)] Schelling, T. S.: (1978), Micromotives and Macrobehavior. W.W. Norton and Co, N.LY.

[Soetevent and Kooreman(2007)] Soetevent, A.R. and Kooreman, P.: (2007), A discrete choice model with social interactions with an application to high school teen behavior, Journal of Applied Econometrics 22(3): 599-624.

[Van Huyck et al.(1990)] Van Huyck, J., Battalio, R., Beil., R.: (1990), Tacit Coordination Games, Strategic Uncertainty and Coordination Failure, The American Economic Review 80(1): 234-248.

[Van Huyck et al.(1997)] Van Huyck, J., Battalio, R., Cook., J.: (1997), Adaptive behavior and coordination failure, Journal of Economic Behavior \& Organization 32(4): 483-503. 